\title{
Neutrino mass hierarchy and matter effects
}

\author{
Alexei Yu. Smirnov* \\ International Center for Theoretical Physics, Trieste, Italy \\ E-mail: smirnoveictp.it
}

\begin{abstract}
Matter effects modify the mixing and the effective masses of neutrinos in a way which depends on the neutrino mass hierarchy. Consequently, for normal and inverted hierarchies the oscillations and flavor conversion results are different. Sensitivity to the mass hierarchy appears whenever the matter effects on the 1-3 mixing and mass splitting become substantial. This happens in supernovae in wide energy range and in the matter of the Earth. The Earth density profile is a multi-layer medium where the resonance and parametric enhancements of oscillations occur. The enhancement is realized in neutrino (antineutrino) channels for the normal (inverted) mass hierarchy. Multi-megaton scale under ice (water) atmospheric neutrino detectors with low energy threshold can establish mass hierarchy with $(3-10) \sigma$ confidence level in few years. The main challenges of these experiments are discussed and various ways to improve sensitivity are outlined. In particular, inelasticity measurements will allow to increase significance of the hierarchy identification by $20-50 \%$.
\end{abstract}

XV Workshop on Neutrino Telescopes,

11-15 March 2013

Venice, Italy

\footnotetext{
*Speaker.
} 


\section{Introduction}

The neutrino mass states can be marked by amount of the electron flavor in such a way that the $v_{e}$ admixture decreases with increase of the state number. The normal mass hierarchy $(\mathrm{NH})$ corresponds then to the ordering 1-2-3, whereas the inverted hierarchy (IH) has the ordering 3-1-2. Therefore "inversion" of the hierarchy means actually the cyclic permutation of the mass states. The hierarchy is related to the sign of $\Delta m_{31}^{2} \equiv m_{3}^{2}-m_{1}^{2}$.

Establishing the mass hierarchy is one of the key objectives in neutrino physics. The mass hierarchy has important phenomenological consequences for the supernova neutrinos, for cosmology, for the atmospheric and accelerator neutrinos propagating in the matter of the Earth. The hierarchy is important whenever the matter effects on oscillations or conversion driven by the 1-3 mixing and mass splittings are important [1]. The strongest effect is in the resonance region where

$$
E \approx \frac{\Delta m_{31}^{2}}{2 V}
$$

and $V$ is the matter potential. In particular, for the Earth matter density the relevant interval is $E \sim(4-10) \mathrm{GeV}$.

From theoretical point of view the NH case is closer to the situation in the quark sector with certain rescaling of mixing. $\mathrm{NH}$ can be easily obtained from the seesaw and with $\mathrm{NH}$ realization of the quark - lepton symmetry and unification is simpler. In contrast, the IH implies strong degeneracy of the two mass states with $\Delta m_{21} / m_{1} \leq \Delta m_{21}^{2} /\left(2 \Delta m_{31}^{3}\right) \leq 1.6 \cdot 10^{-2}$. This, in turn, indicates certain flavor symmetry. In the first approximation the mass spectrum can be considered as a combination of one pseudo-Dirac neutrino and one Majorana neutrino. The former implies maximal 1-2 mixing and substantial deviation from maximal one follows from the charged leptons.

The race for the mass hierarchy has been started. The idea is to explore the matter effects on the 1-3 mixing. This can be realized by studying propagation of high energy neutrinos inside the Earth (see, e.g. [2]) . The possibilities include (i) Detection of atmospheric neutrino fluxes with magnetized spectrometers like INO [3] or with huge atmospheric neutrino detectors PINGU [4], ORCA [5], HyperKamiokande [6]; (ii) Long base-line (LBL) experiments NOvA [7], LBNE [8], LBNO [9]. The ultimate proposal here could be the LBL experiment Fermilab - PINGU [10] for which neutrinos will cross the core of the Earth and undergo the parametric enhancement of oscillations; (iii) detection of low energy supernova neutrinos.

The paper is organized as follows. We first (sec. 2) consider mixing and level splitting in matter and their dependence on the mass hierarchy. Signatures of two hierarchies in supernova neutrinos will be summarized in sec. 3. Sec. 4 is devoted to effects of neutrino propagation in the matter of the Earth. A possibility to establish the hierarchy with huge atmospheric neutrino detectors will de discussed in sec. 5. Different ways to improve sensitivity of these detectors to the hierarchy will be presented in sec. 6 . Sec. 7 summarizes the results.

\section{Mixing and level splitting in matter}

The mixing is determined with respect to the flavor states $v_{f}=\left(v_{e}, v_{\mu}, v_{\tau}\right)$, and mixing matrix connects the flavor states with the eigenstates of the Hamiltonian. Thus, the vacuum mixing 


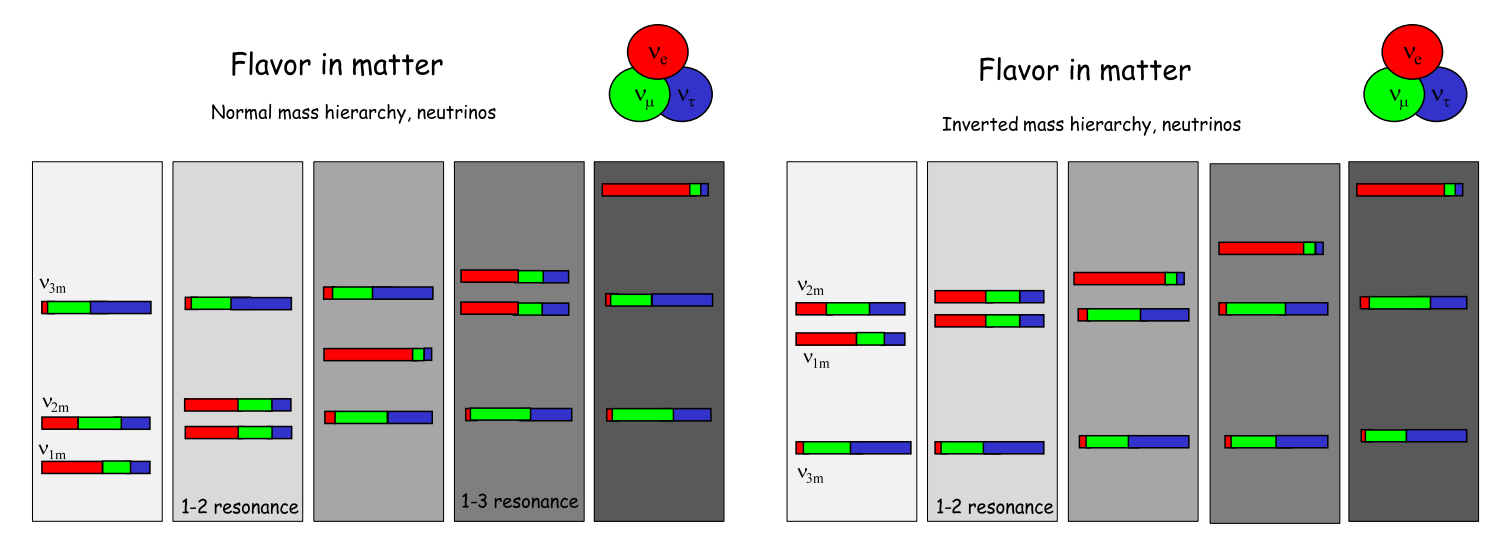

Figure 1: Dependence of the neutrino mass and flavor spectrum on matter density for NH (left panel), IN (right panel). The density increases from the left to right.

connects the flavor states with the mass states: $v_{f}=U_{P M N S} v_{\text {mass }}$, whereas the mixing in matter connects the flavor states with the eigenstates of the Hamiltonian in matter: $v_{f}=U_{P M N S}^{m} \nu_{H}$.

The mixing (flavor composition of the eigenstates) and the level splitting depend on density of matter and neutrino energy, and these dependences are different for NH and IN. Furthermore, the mass and flavor spectra change differently for neutrinos and antineutrinos. In Fig. 1 we show the neutrino spectra for different densities in the cases of normal (left) and inverted (right) hierarchies. For antineutrinos the spectra are shown in Fig. 2. In vacuum spectra for neutrinos and antineutrinos differ for non-zero $\delta_{C P}$. Specifically, the distribution of the $v_{\mu}$ and $v_{\tau}$ flavors in the $v_{1}$ and $v_{2}$ are different. Resonances correspond to configuration when two levels have small splitting and equal admixtures of the electron flavor.

With increase of density the electron flavor becomes heavier, so that for NH it shifts from the lightest state to the heaviest one. It passes through two resonances, and at large densities $v_{e}$ concentrates in the heaviest state. For IH the change is less significant: $v_{e}$ "passes" through one resonance only. At very high densities the effective mass and flavor spectra are similar for both hierarchies. They would be the same for maximal 2-3 mixing. The strongest difference is in the range of 1-3 resonance.

In the antineutrino channel with increase of density the electron flavor becomes lighter. It shifts from the highest to the lowest energy level. There is no resonance in the case of $\mathrm{NH}$, whereas for inverted hierarchy the 1-3 resonance is realized and the change is more substantial. Again the spectra for both hierarchies are similar at high densities.

Inversion of the mass hierarchy is related to the neutrino - antineutrino interchange [11]. Indeed, the $v-\bar{v}$ substitution changes sign of the potential: $V \rightarrow-V$. On the other hand, in the $2 v$ - approximation the inversion of the mass hierarchy means $\Delta m^{2} \rightarrow-\Delta m^{2}$. Therefore under simultaneous transformations $N H \rightarrow I H$ and $v \rightarrow \bar{v}$ the quantity $\Delta m_{31}^{2} /(2 E V)$, which determines characteristics of oscillations in matter, is invariant. This means that both mixing and moduli of the oscillation phase do not change. Thus, in the $2 v$ case

$$
P^{N H}=\bar{P}^{I H}, \quad P^{I H}=\bar{P}^{N H} .
$$




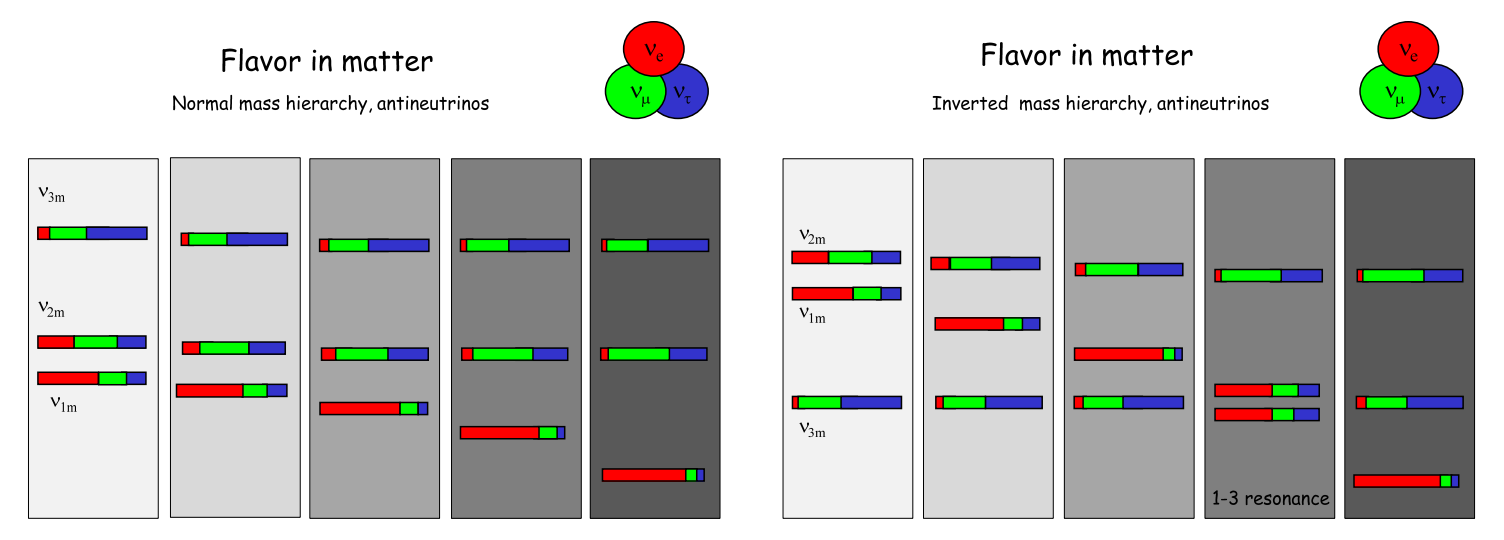

Figure 2: The same as in Fig. 1 for antineutrinos.

Consequently, the difference of numbers of events for NH and IH, $\Delta N \equiv N^{N H}-N^{I H}=\sigma F\left(P^{N H}-\right.$ $\left.P^{I H}\right)$, has opposite sign for neutrinos and antineutrinos:

$$
\Delta \bar{N} \equiv \bar{N}^{N H}-\bar{N}^{I H}=\bar{\sigma} \bar{F}\left(\bar{P}^{N H}-\bar{P}^{I H}\right)=-\bar{\sigma} \bar{F}\left(P^{N H}-P^{I H}\right)=-\frac{\bar{\sigma} \bar{F}}{\sigma F} \Delta N,
$$

where bars indicate characteristics for antineutrinos. Therefore when summed the neutrino and antineutrino signals cancel each other partially because of difference of cross-sections and fluxes for neutrinos and antineutrinos.

Let us consider effect of hierarchy on solar neutrinos. Recall that the 1-2 ordering is normal and this has been established due to matter effects on the 1-2 mass splitting and mixing using the solar neutrinos. Essentially the hierarchy is fixed by the fact that suppression of the neutrino flux in the high energy part of the spectrum is weaker than in the low energy part. For the survival probability we have for low energies $P_{e e} \approx 0.5 \sin ^{2} 2 \theta_{12} \approx 0.58$ and for high energies:

$$
P_{e e}=\left|U_{e 2}\right|^{2} \approx \sin ^{2} \theta_{12} \approx 0.3(N H), \quad P_{e e}=\left|U_{e 1}\right|^{2} \approx \cos ^{2} \theta_{12} \approx 0.66 \quad(I H) .
$$

The solar neutrinos have low sensitivity to the 1-3 ordering because of small matter effect on the 1-3 mixing: $\sin \theta_{13}^{m} \approx \sin \theta_{13}\left(1 \pm\left(2 E V / \Delta m_{31}^{2}\right)\right)$; here $+/-$ correspond to $\mathrm{NH} / \mathrm{IH}$. Then the correction to the survival probability due to matter effect on the 1-3 mixing equals [12]

$$
\frac{\Delta P_{e e}}{P_{e e}} \approx \mp 2 \sin ^{2} \theta_{13} \frac{2 E V_{c}}{\Delta m_{31}^{2}},
$$

where $V_{c}$ is the value of potential in the neutrino production region in the Sun. Minus sign corresponds to $\mathrm{NH}$ and the correction increases with energy. For $E=10 \mathrm{MeV}$ we obtain from (2.4) $\Delta P_{e e} \sim 10^{-3}$ which is below the sensitivity of existing experiments on solar neutrinos.

\section{Supernova neutrinos}

For supernova neutrinos the matter effects dominate, and consequently, the type of hierarchy is crucial. With measured value of the 1-3 mixing the level crossing in the H- (high density) 
resonance is highly adiabatic. This removes many ambiguities and the picture of flavor conversion in the MSW resonance region becomes very simple. Adiabaticity can be broken in shock wavefront only. Results of the MSW conversion can be affected also by the collective neutrino effects that happen in the deeper regions of a star.

The following hierarchy-sensitive effects can be observed.

1) Shock wave breaks adiabaticity of the flavor conversion in the 1-3 resonance. This leads to softening of the spectrum of the electron neutrinos since $v_{e} \rightarrow v_{\mu, \tau}$ conversion becomes less efficient in certain energy interval. The interval shifts with time (during the burst) from low to high energies [13]. Observation of this effects in the neutrino (antineutrino) channel will imply normal (inverted) hierarchy.

2) Neutrino collective effects are more profound in the IH case and can be realized when neutrino density near the core of a star becomes comparable or larger than usual density. If the spectral splits are observed at high energies, the hierarchy should be inverted [14], [15].

3) Sharp time-rise of the $\bar{v}_{e}$ flux and signal in the initial phase of neutrino burst will testify for IH [16].

4) Strong suppression of the $v_{e}$ - neutronization peak is the signature of NH. In this case $v_{e} \rightarrow v_{3}$ transition occurs and the $v_{e}$ survival probability equals $P_{e e}=\sin ^{2} \theta_{13} \approx 0.02$, as compared to $P=\cos ^{2} \theta_{12} \approx 0.68$ in the case of IH [17].

5) At the accretion and cooling phases a strength of partial permutation of the electron and non-electron neutrino spectra depends on the type of mass hierarchy. As a result, the $v_{e}$ energy spectrum (and similarly the $\bar{v}_{e}$ spectrum) becomes two-component: a mixture of the original $v_{e}$ and $v_{\mu}$ spectra. Precise composition depends on the mass hierarchy [17], [18].

6) The Earth matter effects consist of an oscillatory modulation of the neutrino energy spectrum and difference of signals in detectors situated in different places of the Earth [17], [19]. These effects are due to the 1-2 mixing, however their appearance depends on conversion in a star driven by the 1-3 mixing. Being observed in the antineutrino channel the effects will be the evidence of $\mathrm{NH}$, if this happens in the neutrino channel, IH is established.

The problem here is that in the antineutrino channel, which is the most suitable for detection, the difference of original fluxes of the electron and non-electron antineutrinos, and consequently, the oscillation effects are small.

\section{Propagation in the Earth}

Inside the Earth we deal with oscillations in multi-layer medium. The oscillation effects are best presented in the oscillograms - lines of equal oscillation probabilities in the $\left(\cos \theta_{z}-E\right)$ plane, where $\theta_{z}$ is the zenith angle of neutrino trajectory [20], [21] (see Fig. 3 from [22]). The oscillograms depend on mass hierarchy and inverting the hierarchy means approximately an interchange of the $v$ and $\bar{v}$ channel, especially at high energies. Thus, propagation of neutrinos with $E \sim(3-20)$ $\mathrm{GeV}$ can be used to establish the hierarchy. According to Fig. 3 constructed for neutrinos, salient features of the oscillograms are

- the resonance peak (for $P_{e \mu}, P_{e \tau}, P_{\mu e}$ ) or dip (for $P_{e e}$ ) in the mantle domain ( $\cos \theta_{z}>-0.83$ ) at $E \sim 6 \mathrm{GeV}$ and $\cos \theta_{z}=-0.8$; 

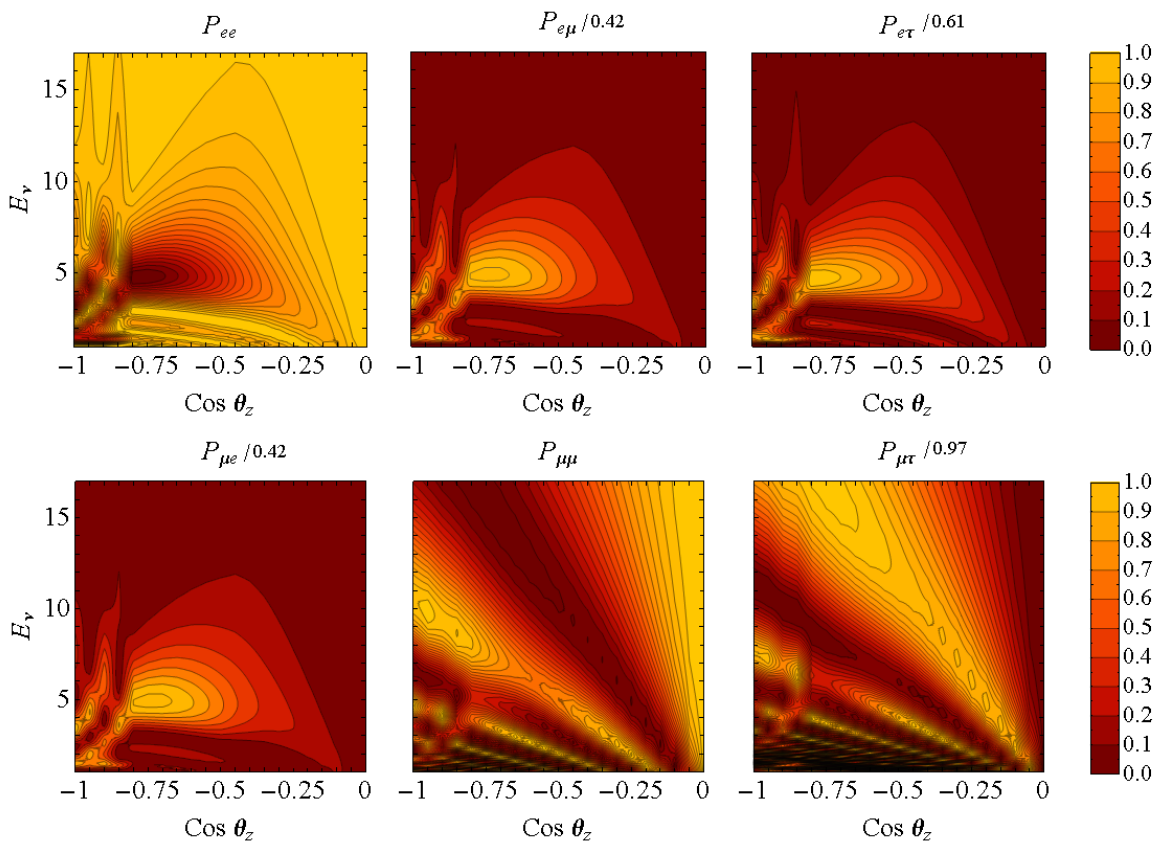

Figure 3: Neutrino oscillograms of the Earth for different oscillation channels for normal mass hierarchy. Shown are the oscillation probabilities normalized by their maximal values (from [22]).

\section{Graphic representation}

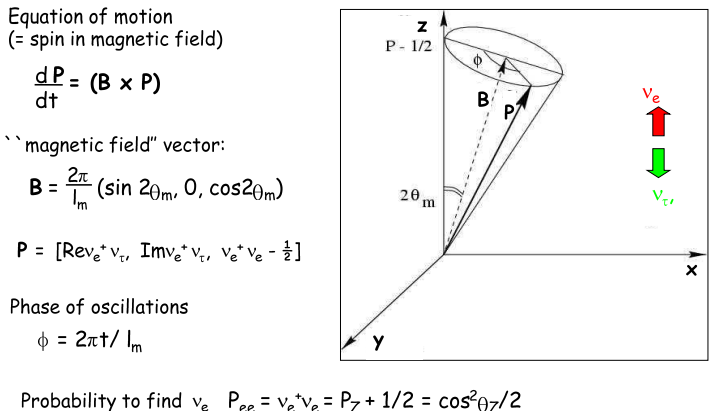

Resonance enhancement in mantle

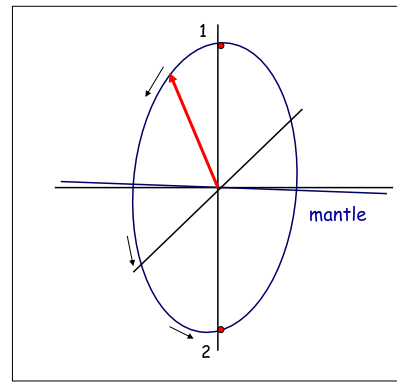

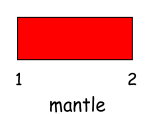

mantle

Figure 4: Graphic representation of the neutrino oscillations. Left panel: generic case with explanations; right panel: motion of the neutrino vector which corresponds to the peak in oscillogram due to the resonance enhancement of the $v_{e}-v_{\tau}^{\prime}$ oscillations in mantle. The oscillation phase equals $\pi$.

- three parametric ridges in the core domain $\left(\cos \theta_{z}<-0.83\right)$ at $E_{V}=(2-10) \mathrm{GeV}$.

The most transparent and easiest way to understand these effects is to use graphic representation based on analogy of the neutrino oscillations with the electron spin precession in the magnetic field (see Fig. 4, left). (For definiteness we consider the two neutrino system, $v_{e}$ and $v_{\tau}^{\prime}$, with $v_{\tau}^{\prime}$ being certain mixture of $v_{\mu}$ and $v_{\tau}$.) Precession of the neutrino vector $\mathbf{P}$ leads to periodic change of its projection onto axis $z$, which is equivalent to oscillations. 
Parametric enhancement in the 1-3 mode

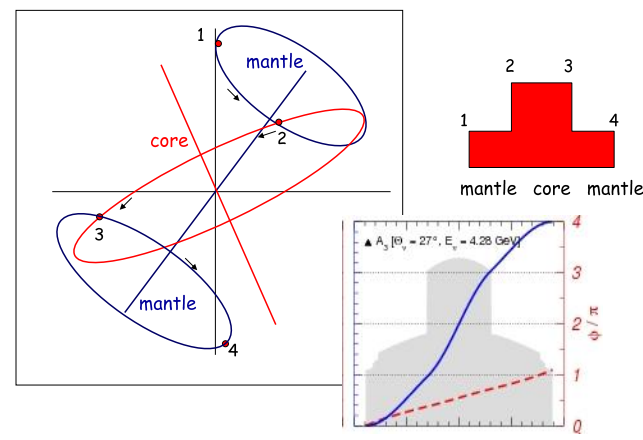

Parametric enhancement in the 1-2 mode

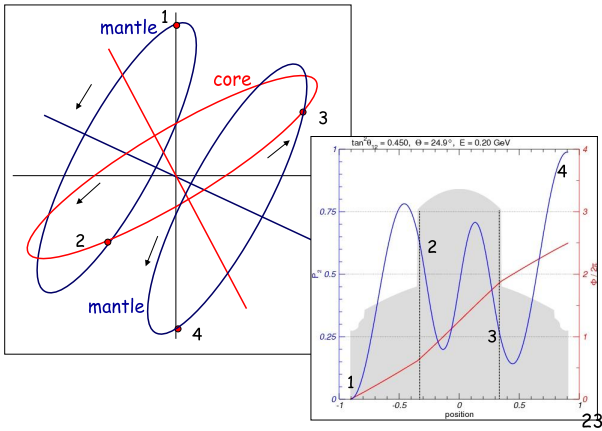

Figure 5: Graphic representation of the parametric enhancement of the $v_{e}-v_{\tau}^{\prime}$ oscillations. Left panel: motion of the neutrino vector which corresponds to the parametric ridge in oscillogram due to the 1-3 mixing. In the lower box shown is the probability as function of distance (from [21]). Right panel: the same as in the left panel but for oscillations driven by the 1-2 mixing.

In Figs. 4, 5 we show graphic representations of the main effects involved in propagation of neutrinos inside the Earth:

1. Resonance enhancement of oscillations (Fig. 4, right). The MSW resonance condition is fulfilled in the mantle at $6 \mathrm{GeV}$. In resonance $2 \theta_{m}=\pi / 2$, so that the cone axis is directed along the axis $x$ and precession occurs with maximal amplitude. For the zenith angle $\left|\cos \theta_{z}\right|=0.8$ the length of trajectory in the mantle is such that $L=l_{m} / 2$ and therefore the oscillation phase equals $\pi$, so that in final state $\mathbf{P}_{z}=-1 / 2$. This corresponds to complete flip of $\mathbf{P}$ and maximal probability of the $v_{e} \rightarrow v_{\tau}^{\prime}$ transition. and happens in the peak in the oscillogram at $E=6 \mathrm{GeV}$.

2. Parametric enhancement of oscillations (Fig. 5) occur for the core crossing trajectories when neutrinos experience propagation through three layers with slowly changing density and two density jumps on the border between the layers [23]. This leads to the parametric ridges. The cone axis has different directions in the mantle and core (Fig. 5). The axis changes its direction suddenly at the border between the mantle and the core. So, the vector $\mathbf{P}$ precesses in the core and mantle around two different directions. For certain $E$ and $\theta_{z}$ the phases in the core and mantle can be such that the probability of transition builds up to maximal one without return and oscillations (see lower boxes). This produces strong transition even if in each layer the transition is small. Two such possibilities are shown in Fig. 5.

\section{Hierarchy with Huge atmospheric neutrino detectors}

The atmospheric neutrino fluxes, being cost free, cover complete zenith angle range and huge interval of energies which includes the resonance region relevant for determination of the hierarchy. The original fluxes produced in atmosphere contain $v_{\mu}$ and $v_{e}$ and corresponding antineutrinos. Furthermore, the flavor content changes with the neutrino energy. The main challenges here are (i) uncertainties of the original fluxes; (ii) reconstruction of the neutrino direction; (iii) the neutrino energy resolution; (iv) flavor identification. According to earlier estimations [24] the DeepCore detector with the energy threshold above $10 \mathrm{GeV}$ has rather low sensitivity to the hierarchy. High 

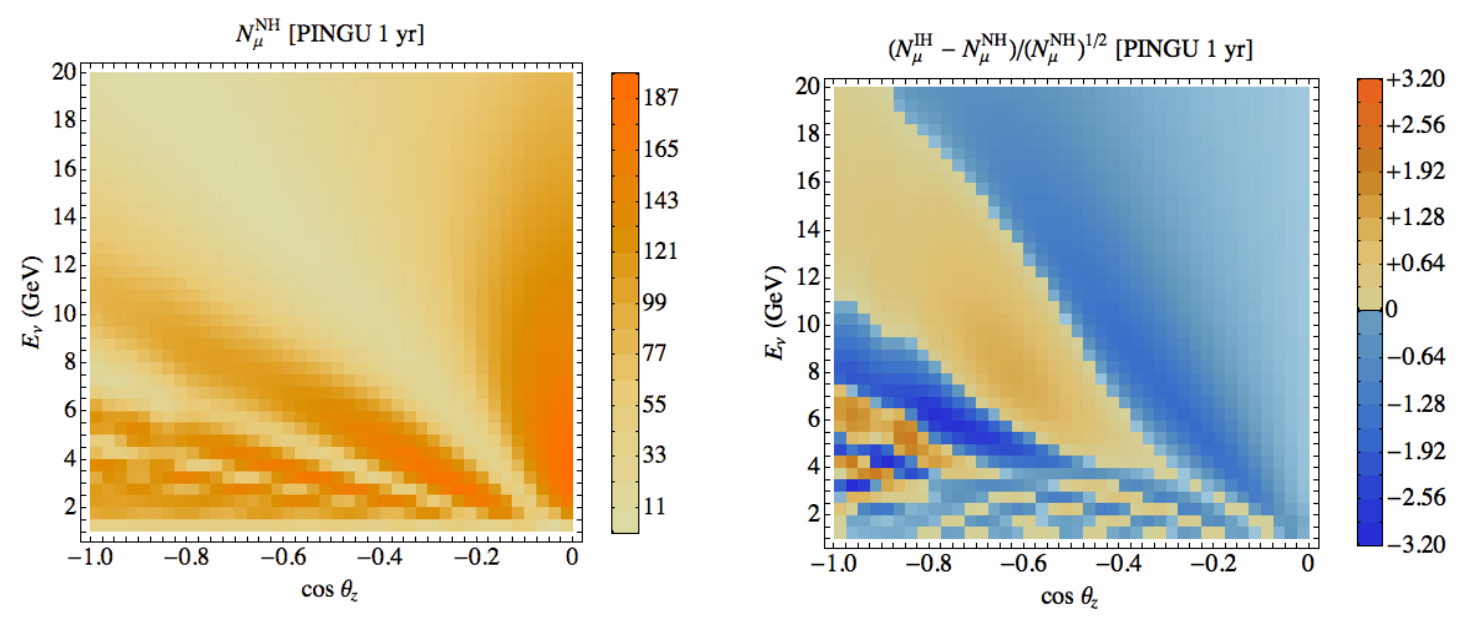

Figure 6: Left panel: The distribution of the $v_{\mu}$ events in the $\left(E_{v}-\cos \theta_{z}\right)$ plane that can be collected by PINGU detector during about 3 years. NH is assumed. Right panel: The hierarchy asymmetry of $v_{\mu}$ events in the $E_{v}-\cos \theta_{z}$ plane without smearing (adapted from [22]).

statistics and even mild technological developments which will be achieved in Multi-megaton under ice (water) cherenkov detectors with relatively low energy threshold $E_{v} \sim(2-3) \mathrm{GeV}$ can resolve these problems [22], [25]. Thus, PINGU detector [4] will have up to $10^{5}$ events in the range (2 20) $\mathrm{GeV}$ which covers the 1-3 resonance region.

Important sample of events is due to the $v_{\mu}$ - charged current interactions (the $\mu$ - track events): $v_{\mu}+n \rightarrow \mu+h$. With densely instrumented detector it is possible to measure the muon energy $E_{\mu}$ and muon direction, $\theta_{\mu}, \phi_{\mu}$, as well as the energy of hadron cascade, $E_{h}$. This becomes possible analyzing time development of the event. Consequently, the neutrino energy, $E_{v}=E_{\mu}+E_{h}$ and, to some extend, the neutrino direction can be reconstructed. In Fig. 6 (left) we show a distribution of the $v_{\mu}$ events in the $E_{v}-\cos \theta_{z}$ plane.

Quick estimation of discovery potential can be obtained using the hierarchy (H-) asymmetry. For each $i j-$ bin in the $\left(E_{v}-\cos \theta_{z}\right)$ plane the H-asymmetry is defined as [22]

$$
S_{i j}=\frac{N_{i j}^{I H}-N_{i j}^{N H}}{\sqrt{N_{i j}^{N H}}} .
$$

If NH is the true hierarchy, $N_{i j}^{N H}$ can be considered as the "experimental" number of events, whereas $N_{i j}^{I H}$ - as the "fit" number of events. Then $\left|S_{i j}\right|$ reflects statistical significance of establishing true hierarchy. Clearly this quantity does not take into account fluctuations and therefore more appropriate term could be distinguishability. Still $S_{i j}$ is very useful characteristic which allows one to study dependence of the discovery potential on values of involved parameters, uncertainties, degeneracies, etc..

The uncorrelated systematic errors can be introduced adding to the denominator of (5.1)

$$
N_{i j}^{N H} \rightarrow \sigma_{i j}^{2}=N_{i j}^{N H}+\left(f N_{i j}^{N H}\right)^{2}
$$



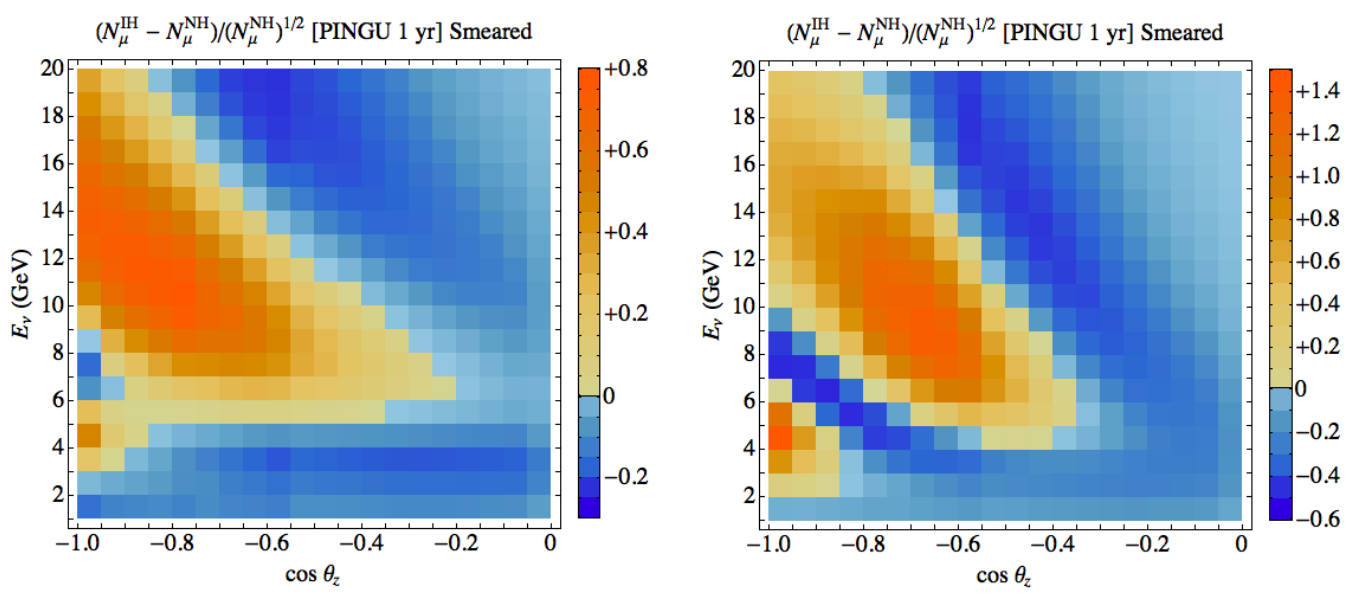

Figure 7: Smeared H-asymmetry distribution of the $v_{\mu}$ events in the $E_{v}-\cos \theta_{z}$ plane for two different values of width of the angular resolution function: $\sigma_{\theta}=\sqrt{m_{p} / E_{v}}\left(\right.$ left panel) and $\sigma_{\theta}=0.5 \sqrt{m_{p} / E_{v}}$ (right panel). We use $\sigma_{E}=0.2 E_{V}$ for the width of the energy reconstruction function (from [22]).

where $f$ determines the level of systematic errors. If measurements in each bin are independent the total significance is then given by

$$
S_{t o t}=\sqrt{\sum_{i j}\left|S_{i j}\right|^{2}}
$$

In Fig. 6 (right) we show the binned distribution of the H-asymmetry. As follows from the figure, there are regions with different signs of asymmetry, and size of these regions increases with energy. To enhance effect, integration over regions with different signs of $S$ should be avoided, which requires good enough energy and angle reconstruction. Reconstruction of the neutrino energy depends on the experimental energy resolution for muon and cascade. Reconstruction of the neutrino direction includes both the experimental and kinematic uncertainties. The latter is related to the scattering angle between muon and neutrino. These uncertainties can be taken into account performing smearing of the distribution with Gaussian functions characterized by (half) widths

$$
\sigma_{E}=A E_{V}, \quad \sigma_{\theta}=B\left(m_{p} / E_{V}\right)
$$

Here $A$ and $B$ are the parameters which can be varied in the intervals $A=0.2-0.3$ and $B=0.3-1.5$, $m_{p}$ is the mass of proton. Total significance is given by formula (5.3) for smeared $S_{i j}$.

In Fig. 7 we show the smeared asymmetries for two different width $\sigma_{\theta}$. Smearing (i) eliminates fine structures, especially at low energies, (ii) shifts the region of high sensitivity to hierarchy to higher energies $(8-15) \mathrm{GeV}$ and larger $\left|\cos \theta_{z}\right|$; (iii) reduces significance in the individual bins, and consequently, total significance. Systematics further reduces significance by factor $\sim 2$ [22].

For parameters $A=0.2$ and $B=1$ (which are rather close to the parameters given by PINGU collaboration) one obtains that with $f=5 \%$ (uncorrelated systematic errors) the $5 \sigma$ distinguishability can be achieved in 2 years. The distinguishability increases up to $7 \sigma$ after 5 years of operation. These computations correspond to rather large effective volume. Optimized volume which implements an additional cut of events by threshold of 21 DOM hits is about 3 time smaller [4] which 
further reduces significance by $\sim \sqrt{3}$. Still $3 \sigma$ distinguishability can be achieved after 5 years under conservative assumptions.

Another problem is degeneracy of the hierarchy effects with other effects and parameters involved. The strongest degeneracy is related to not well known value of $\Delta m_{31}^{2}$. The smeared distribution of quantity $A_{m} \equiv\left[N_{\mu}^{N H}\left(\Delta m_{31}^{2}+1 \sigma\right)-N_{\mu}^{N H}\left(\Delta m_{31}^{2}\right)\right] / N_{\mu}^{N H}\left(\Delta m_{31}^{2}\right)$ is very similar to the Hasymmetry distribution. Furthermore, maximal significance of $A_{m}$ in the individual bins in about 2 times larger [22]. The difference is that region of strong $\Delta m_{31}^{2}$ effect is at larger energies $(13-18)$ $\mathrm{GeV}$ and in vertical direction, $\cos \theta_{z} \approx-1.0$, as compared with $(8-14) \mathrm{GeV}$ and $\cos \theta_{z}=-0.8$ for the mass hierarchy. Unfortunately, NOvA and T2K will not be able to measure $\Delta m_{31}^{2}$ with high enough accuracy to avoid this degeneracy. Essentially, PINGU will have higher sensitivity to $\Delta m_{31}^{2}$. This means that in analysis of the PINGU data one needs to consider $\Delta m_{31}^{2}$ as free fit parameter along with the mass hierarchy. The $\Delta m_{31}^{2}-$ dependent asymmetry,

$$
S_{i j}^{2}\left(\Delta m_{31, f i t}^{2}\right)=\left[N_{i j}^{N H}\left(\Delta m_{31, f i t}^{2}\right)-N_{i j}^{N H}\left(\Delta m_{31, \text { true }}^{2}\right)\right]^{2} / \sigma_{i j}^{2},
$$

should be minimized with respect to $\Delta m_{31, f i t}^{2}$. In the minimum of $S_{t o t}^{2}\left(\Delta m_{31}^{2}\right)$ the shift of mass squared difference equals $\left(\Delta m_{31, \text { fit }}^{2}-\Delta m_{31, \text { true }}^{2}\right) \sim 5 \cdot 10^{-5} \mathrm{eV}^{2}$ which is about $0.5 \sigma$ only [22]. So, distinguishability is reduced, e.g., from $6 \sigma$ (for fixed $\Delta m_{31}^{2}$ ) down to $3.8 \sigma$, i.e. by $37 \%$ [22].

\section{Improving sensitivity to mass hierarchy}

The main challenges for huge detectors are

(i) Flavor identification: in particular, identification of the $v_{\mu}$ - events in view of additional contribution of the $v_{\tau}-\tau-\mu$ events, the $\mu-\pi$ misidentification, contamination of the $v_{\mu}$ - sample by the CC $v_{e}$ and the neutral current interactions, etc..

(ii) Smearing over energies and directions. This includes both kinematic smearing, that is, integration over the angle between the neutrino and muon, and the experimental smearing.

(iii) Degeneracy of parameters related to uncertainties in $\Delta m_{31}^{2}, \theta_{23}, \delta$. Degeneracy of hierarchy with $\delta$ is small [22]: the effect of $\delta$ is substantially smaller, and furthermore, it is mainly at low energies.

(iv) Systematics.

Cancellation of the $v$ and $\bar{v}$ hierarchy asymmetries reduces the total significance. As we have mentioned, if the $v$ and $\bar{v}$ signals are not separated, they partially cancel each other in the total significance:

$$
S^{t o t} \approx\left|S_{V}\right|-\left|\bar{S}_{v}\right|
$$

In the case when $v$ and $\bar{v}$ signals are measured separately, one would have

$$
S^{t o t} \approx \sqrt{S_{v}^{2}+z \bar{S}_{v}^{2}}
$$

where $z=0(1)$ depends on the ratio of numbers of $v$ and $\bar{v}$ events. The ideal separation enhances the significance by factor $2-3$. However, due to finite accuracy of separation, improvement of significance is by factor $1.1-1.2$ only [26].

There are different ways to improve sensitivity to hierarchy. 

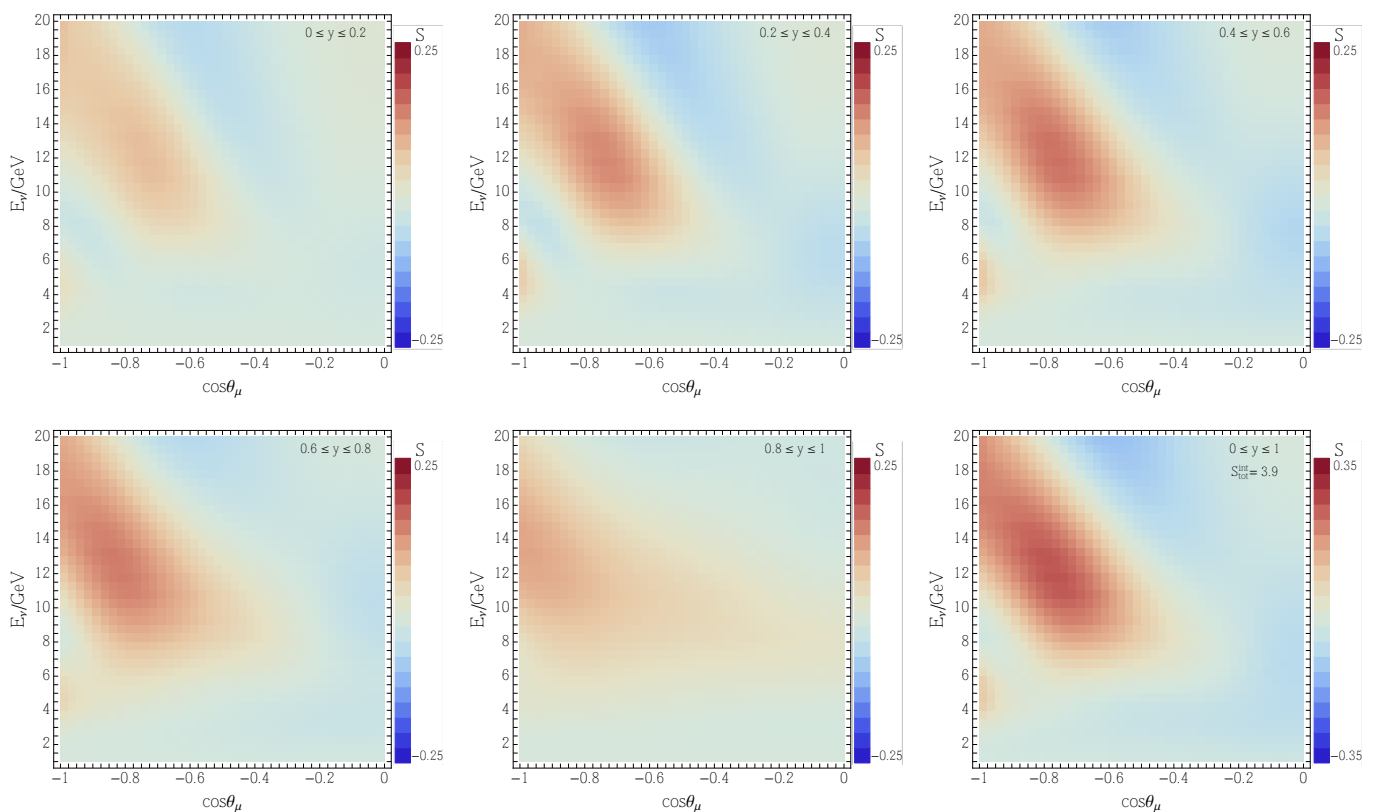

Figure 8: Smeared hierarchy asymmetry distribution of the $v_{\mu}$ events in the $E_{v}-\cos \theta_{z}$ plane for different intervals of $y$. The last panel (bottom, right) shows the $y$-integrated distribution (from [26]).

1) Use the inelasticity ( $y$ - distribution) in addition to $E_{v}$ and $\theta_{\mu}$, that is, the 3 dimensional distributions of events over $\left(E_{v}, c_{\mu}, y\right)$ or $\left(E_{\mu}, E_{h}, c_{\mu}\right)$, here $c_{\mu} \equiv \cos \theta_{\mu}$.

2) Include other types of events in analysis. In particular, the $v_{e}$ events have high sensitivity to the hierarchy [22], [27]. The problem here is that these events have bad angular resolution and they are contaminated by the neutral current interactions of all flavors, by the $v_{\tau}$ events, as well as by the $v_{\mu}$ events with faint muons or muon misidentified with pion.

3) Direction of cascades. Already $90^{\circ}-180^{\circ}$ accuracy of the determination would lead to substantial improvement of sensitivity to the hierarchy of both $v_{e}$ and $v_{\mu}$ events.

Measurements of the inelasticity, i.e. fraction of the total energy transferred to hadrons,

$$
y=\frac{E_{v}-E_{\mu}}{E_{v}}
$$

open up a possibility of [26]:

- Partial separation (on statistical ground) of the neutrino and antineutrino signals, since

$$
N_{v} \sim 1, \quad N_{v} \sim(1-y)^{2} .
$$

Fitting the measured $y$ distribution for each bin allows one to extract fractions of $v$ and $\bar{v}$.

- Better reconstruction of the neutrino direction, and therefore reduction of the kinematic smearing: Indeed, the angle between neutrino and muon, $\beta$, is determined by

$$
\sin ^{2} \beta / 2=\frac{x y m_{N}}{2 E_{\mu}},
$$

where $x$ is the Bjorken variable. Selection of events with small $y$ reduces possible values of $\beta$. 
- Better control over systematics.

- Reduction of degeneracy. In fact, this gives the largest gain in significance [26].

In Fig. 8 we show the $\mathrm{H}$-asymmetries for different intervals of $y$. Two improvements, $v-\bar{v}$ separation and reduction of the kinematic smearing anticorrelate: For small $y$ the angular distribution is good, but the $v-\bar{v}$ separation is bad. On the contrary, for large $y$ the $v-\bar{v}$ separation improves, but the angular resolution worsens. As a result, the largest contribution to the significance comes from the intermediate region of $y$. Without loss of information and introduction of new systematics both effects are taken into account automatically if one uses immediately the 3D distribution of events. According to Fig. 8 with increase of $y$ the region of highest sensitivity shifts to larger energies and $\left|\cos \theta_{z}\right|$. The change of distribution with $y$ allows one to reduce systematics.

The distribution of events over $E_{v}, c_{\mu}, y$ is given by [26]

$$
n\left(E_{v}, c_{\mu}, y\right)=\frac{1}{\pi} \int d c_{\mu} \rho\left(E_{v}, c_{v}\right) g\left(E_{v}, c_{v}, c_{\mu}, y\right),
$$

where

$$
\rho\left(E_{v}, c_{v}\right)=2 \pi N_{A} n_{i c e} V_{e f f} T \Phi_{\mu}\left[P_{\mu \mu}-r^{-1} P_{e \mu}\right]
$$

is the density of the neutrino events, $c_{v} \equiv \cos \theta_{z}, r=\Phi_{\mu} / \Phi_{e}$ is the ratio of $v_{\mu}$ and $v_{e}$ fluxes, $n_{i c e}$ is the density of ice, $N_{A}$ is the Avogadro number, $V_{e f f}$ is the effective volume of detector, $T$ is the exposure time. Here

$$
g\left(E_{v}, c_{v}, c_{\mu}, y\right)=\int d x \frac{d^{2} \sigma}{d x d y} \frac{1}{\sqrt{\left(s_{\mu} s_{\beta}\right)^{2}-\left(c_{v}-c_{\mu} c_{\beta}\right)^{2}}},
$$

can be considered as the kinematic smearing function. For antineutrinos one needs to change fluxes and the probabilities correspondingly. The probabilities (as well as the effective volumes) are different for $\mathrm{NH}$ and $\mathrm{IH}$. Then the total number of events is given by $n=n+\bar{n}$. All these distributions should be smeared over the experimental resolution functions. Total significance of events is given by

$$
\left|S_{t o t}\right|=\left\{\int d c_{\mu} \int d E_{v} \int d y \frac{\left[n^{I H}\left(E_{v}, c_{\mu}, y\right)-n^{N H}\left(E_{v}, c_{\mu}, y\right)\right]^{2}}{n^{N H}\left(E_{v}, c_{\mu}, y\right)}\right\}^{1 / 2},
$$

if the $y$-distribution is taken into account. Here summation over bins is substituted by integration. Without y-distribution (y-integrated) we would have

$$
\left|S_{\text {tot }}^{\text {int }}\right|=\left\{\int d c_{\mu} \int d E_{v} \frac{\left(\int d y\left[n^{I H}\left(E_{v}, c_{\mu}, y\right)-n^{N H}\left(E_{v}, c_{\mu}, y\right)\right]\right)^{2}}{\int d y n^{N H}\left(E_{v}, c_{\mu}, y\right)}\right\}^{1 / 2} .
$$

Notice that in Eq. (6.10) the density of events is integrated over $y$ before computing the significance (under squared).

Results on the total significance (distinguishability) which can be obtained during 1 year of exposure (for the effective volume from [22]) can be summarized in the following way. Without experimental smearing we would have $S_{t o t}=6.4$. Degeneracy reduces this number down to $S_{t o t}=$ 3.6. The use of inelasticity increases it up to 4.8 . The experimental smearing with $\sigma_{E}=\left(0.7 E_{v}\right)^{0.5}$ and $\sigma_{\psi}=20^{\circ} \sqrt{m_{p} / E_{\mu}}$ reduces these numbers as $3.54 \rightarrow 1.90 \rightarrow 2.17$. 


\section{Conclusion}

1. Interaction with matter changes the neutrino mixing and effective mass splitting in a way that depends on the mass hierarchy. Consequently, results of oscillations and flavor conversion are different for the two hierarchies.

2. Sensitivity to the mass hierarchy appears whenever the matter effect on the 1-3 mixing and mass splitting becomes substantial. This happens in supernovae in large energy range, and in the matter of the Earth.

3. The Earth density profile is a multi-layer medium where the resonance enhancement of oscillations as well as the parametric enhancement of oscillations occur. The enhancement is realized in neutrino (antineutrino ) channels for normal (inverted) mass hierarchy.

4. Multi-megaton scale under ice (water) atmospheric neutrino detectors with low energy threshold $(2-3 \mathrm{GeV})$ may establish mass hierarchy with $\sim(3-10) \sigma$ confidence level in few years.

5. The main challenges of these experiments are flavor identification of events, accuracy of measurements of energies and directions, systematics, degeneracy of parameters.

6. There are various ways to improve the sensitivity to the hierarchy. This includes in particular, consideration of the $v_{e}$ events, reconstruction of the cascade direction, etc.. Inelasticity measurements will allow to increase significance of the hierarchy identification by $20-50 \%$.

7. Detection of a neutrino burst from relatively close supernovae (which may occur any time) may resolve the issue of neutrino mass hierarchy.

\section{References}

[1] M. Blennow and A. Y. Smirnov, Neutrino propagation in matter, Adv. High Energy Phys. 2013 (2013) 972485 [arXiv:1306.2903 [hep-ph]].

[2] J. Bernabeu, S. Palomares Ruiz and S. T. Petcov, Atmospheric neutrino oscillations, theta(13) and neutrino mass hierarchy, Nucl. Phys. B 669 (2003) 255 [hep-ph/0305152]; D. Indumathi and M. V. N. Murthy, A Question of hierarchy: Matter effects with atmospheric neutrinos and anti-neutrinos, Phys. Rev. D 71 (2005) 013001 [hep-ph/0407336]; S. Palomares-Ruiz and S. T. Petcov, Three-neutrino oscillations of atmospheric neutrinos, theta(13), neutrino mass hierarchy and iron magnetized detectors, Nucl. Phys. B 712 (2005) 392 [hep-ph/0406096]; R. Gandhi, P. Ghoshal, S. Goswami, P. Mehta and S. U. Sankar, Earth matter effects at very long baselines and the neutrino mass hierarchy, Phys. Rev. D 73 (2006) 053001 [hep-ph/0411252]; S. T. Petcov and T. Schwetz, Determining the neutrino mass hierarchy with atmospheric neutrinos, Nucl. Phys. B 740 (2006) 1 [hep-ph/0511277]; R. Gandhi, P. Ghoshal, S. Goswami, P. Mehta, S. U. Sankar and S. Shalgar, Mass Hierarchy Determination via future Atmospheric Neutrino Detectors, Phys. Rev. D 76 (2007) 073012 [arXiv:0707.1723 [hep-ph]]; V. Barger, R. Gandhi, P. Ghoshal, S. Goswami, D. Marfatia, S. Prakash, S. K. Raut and S U. Sankar, Neutrino mass hierarchy and octant determination with atmospheric neutrinos, Phys. Rev. Lett. 109 (2012) 091801 [arXiv:1203.6012 [hep-ph]].

[3] INO, India-Based Neutrino Observatory http://www.ino.tifr.res.in/ino/. M. Blennow and T. Schwetz, Identifying the Neutrino mass Ordering with INO and NOvA, JHEP 1208 (2012) 058 [Erratum-ibid. 1211 (2012) 098] [arXiv:1203.3388 [hep-ph]]; A. Ghosh, T. Thakore and S. Choubey, Determining the Neutrino Mass Hierarchy with INO, T2K, NOvA and Reactor Experiments, JHEP 1304 (2013) 009 
[arXiv:1212.1305]; A. Samanta, Discrimination of mass hierarchy with atmospheric neutrinos at a magnetized muon detector, Phys. Rev. D 81 (2010) 037302 [arXiv:0907.3540 [hep-ph]].

[4] M. G. Aartsen et al. [IceCube and PINGU Collaborations], PINGU Sensitivity to the Neutrino Mass Hierarchy, arXiv:1306.5846 [astro-ph.IM], D. J. Koskinen, IceCube-DeepCore-PINGU: Fundamental neutrino and dark matter physics at the South Pole, Mod. Phys. Lett. A 26 (2011) 2899.

[5] P. Coyle et al. Km3Net Contribution to the European Strategy Preparatory Group Symposium. September 2012, Krakow, Poland.

[6] K. Abe, T. Abe, H. Aihara, Y. Fukuda, Y. Hayato, K. Huang, A. K. Ichikawa and M. Ikeda et al., Letter of Intent: The Hyper-Kamiokande Experiment - Detector Design and Physics Potential -, arXiv:1109.3262 [hep-ex].

[7] R. B. Patterson [NOvA Collaboration], The NOvA Experiment: Status and Outlook, Nucl. Phys. Proc. Suppl. 235-236 (2013) 151 [arXiv:1209.0716 [hep-ex]]; O. Mena, H. Nunokawa and S. J. Parke, NOvA and T2K: The Race for the neutrino mass hierarchy, Phys. Rev. D 75 (2007) 033002 [hep-ph/0609011].

[8] J. M. Paley [NOvA and LBNE Collaborations], The search for CP violation and the determination of the neutrino mass hierarchy in NOvA and LBNE, PoS ICHEP 2012 (2013) 393.

[9] A. Stahl, C. Wiebusch, A. M. Guler, M. Kamiscioglu, R. Sever, A. U. Yilmazer, C. Gunes and D. Yilmaz et al., Expression of Interest for a very long baseline neutrino oscillation experiment (LBNO), CERN-SPSC-2012-021.

[10] J. Tang and W. Winter, Requirements for a New Detector at the South Pole Receiving an Accelerator Neutrino Beam, JHEP 1202 (2012) 028 [arXiv:1110.5908 [hep-ph]].

[11] H. Minakata, H. Nunokawa and S. J. Parke, CP and T violation in neutrino oscillations, AIP Conf. Proc. 670 (2003) 132 [hep-ph/0306221] (see also [22]).

[12] S. Goswami and A. Y. Smirnov, Solar neutrinos and 1-3 leptonic mixing, Phys. Rev. D 72 (2005) 053011 [hep-ph/0411359].

[13] M. Kachelriess and R. Tomas, Identifying the neutrino mass hierarchy with supernova neutrinos, [hep-ph/0412100]; V. Barger, P. Huber and D. Marfatia, Supernova neutrinos can tell us the neutrino mass hierarchy independently of flux models, Phys. Lett. B 617 (2005) 167 [hep-ph/0501184].

[14] H. Duan, G. M. Fuller, J. Carlson and Y. -Q. Zhong, Neutrino Mass Hierarchy and Stepwise Spectral Swapping of Supernova Neutrino Flavors, Phys. Rev. Lett. 99 (2007) 241802 [arXiv:0707.0290 [astro-ph]]; B. Dasgupta, A. Mirizzi, I. Tamborra and R. Tomas, Neutrino mass hierarchy and three-flavor spectral splits of supernova neutrinos, Phys. Rev. D 81 (2010) 093008 [arXiv:1002.2943 [hep-ph]].

[15] B. Dasgupta, A. Dighe, G. G. Raffelt and A. Y. Smirnov, Phys. Rev. Lett. 103 (2009) 051105 [arXiv:0904.3542 [hep-ph]].

[16] P. D. Serpico, S. Chakraborty, T. Fischer, L. Hudepohl, H. -T. Janka and A. Mirizzi, Probing the neutrino mass hierarchy with the rise time of a supernova burst, Phys. Rev. D 85 (2012) 085031 [arXiv:1111.4483 [astro-ph.SR]].

[17] A. S. Dighe and A. Y. Smirnov, Identifying the neutrino mass spectrum from the neutrino burst from a supernova, Phys. Rev. D 62 (2000) 033007 [hep-ph/9907423].

[18] C. Lunardini and A. Y. Smirnov, Probing the neutrino mass hierarchy and the 13 mixing with supernovae, JCAP 0306 (2003) 009 [hep-ph/0302033]. 
[19] C. Lunardini and A. Y. Smirnov, Supernova neutrinos: Earth matter effects and neutrino mass spectrum, Nucl. Phys. B 616 (2001) 307 [hep-ph/0106149]; A. S. Dighe, M. T. Keil and G. G. Raffelt, Detecting the neutrino mass hierarchy with a supernova at IceCube, JCAP 0306 (2003) 005 [hep-ph/0303210].

[20] P. Lipari, (1998) unpublished; T. Ohlsson and H. Snellman, Neutrino oscillations with three flavors in matter: Applications to neutrinos traversing the Earth, Phys. Lett. B 474 (2000) 153 [hep-ph/9912295]; T. Kajita Atmospheric neutrinos, New J. Phys. 6 (2004) 194.

[21] E. K. Akhmedov, M. Maltoni and A. Y. Smirnov, Neutrino oscillograms of the Earth: Effects of 1-2 mixing and CP-violation, JHEP 0806 (2008) 072 [arXiv:0804.1466 [hep-ph]]; E. K. Akhmedov, M. Maltoni and A. Y. Smirnov, 1-3 leptonic mixing and the neutrino oscillograms of the Earth, JHEP 0705 (2007) 077 [hep-ph/0612285].

[22] E. K. Akhmedov, S. Razzaque and A. Y. Smirnov, Mass hierarchy, 2-3 mixing and CP-phase with Huge Atmospheric Neutrino Detectors, JHEP 1302 (2013) 082 [JHEP 1302 (2013) 082] [Erratum-ibid. 1307 (2013) 026] [arXiv:1205.7071 [hep-ph]].

[23] Q. Y. Liu and A. Y. Smirnov, Neutrino mass spectrum with muon-neutrino $\rightarrow$ sterile-neutrino oscillations of atmospheric neutrinos, Nucl. Phys. B 524 (1998) 505 [hep-ph/9712493]. S. T. Petcov, Diffractive - like (or parametric resonance - like?) enhancement of the earth (day - night) effect for solar neutrinos crossing the earth core, Phys. Lett. B 434 (1998) 321 [hep-ph/9805262].

E. K. Akhmedov, Parametric resonance of neutrino oscillations and passage of solar and atmospheric neutrinos through the earth, Nucl. Phys. B 538 (1999) 25 [hep-ph/9805272]. E. K. Akhmedov, A. Dighe, P. Lipari and A. Y. Smirnov, Atmospheric neutrinos at Super-Kamiokande and parametric resonance in neutrino oscillations, Nucl. Phys. B 542 (1999) 3 [hep-ph/9808270].

[24] O. Mena, I. Mocioiu and S. Razzaque, Neutrino mass hierarchy extraction using atmospheric neutrinos in ice, Phys. Rev. D 78 (2008) 093003 [arXiv:0803.3044 [hep-ph]].

[25] W. Winter, Neutrino mass hierarchy determination with IceCube-PINGU, Phys. Rev. D 88 (2013) 013013 [arXiv:1305.5539 [hep-ph]]. D. Franco, C. Jollet, A. Kouchner, V. Kulikovskiy, A. Meregaglia, S. Perasso, T. Pradier and A. Tonazzo et al., Mass hierarchy discrimination with atmospheric neutrinos in large volume ice/water Cherenkov detectors, JHEP 1304 (2013) 008 [arXiv:1301.4332 [hep-ex]].

[26] M. Ribordy and A. Y. Smirnov, Improving the neutrino mass hierarchy identification with inelasticity measurement in PINGU and ORCA, Phys. Rev. D 87 (2013) 113007 [arXiv:1303.0758 [hep-ph]].

[27] J. Brunner, Counting Electrons to Probe the Neutrino Mass Hierarchy, arXiv:1304.6230 [hep-ex]. 